\title{
A Matched Prospective Study of Human Immunodeficiency Virus Serostatus, Human Papillomavirus DNA, and Cervical Lesions Detected by Cytology and Colposcopy
}

\author{
L.O. Eckert, ${ }^{1 *}$ D.H. Watts, ${ }^{1}$ L.A. Koutsky, ${ }^{2}$ S.E. Hawes, ${ }^{2,3}$ \\ C.E. Stevens, ${ }^{1}$ J. Kuypers, ${ }^{3}$ and N.B. Kiviat ${ }^{3}$ \\ ${ }^{1}$ Department of Obstetrics and Gynecology, University of Washington, Seattle \\ ${ }^{2}$ Department of Epidemiology, University of Washington, Seattle \\ ${ }^{3}$ Department of Pathology, University of Washington, Seattle
}

\begin{abstract}
Objective: To compare the prevalence and type of human papillomavirus (HPV) infections in the genital tract of human-immunodeficiency-virus- (HIV) seropositive and -seronegative women matched for cytology and to examine prospectively the relationship of HPV DNA, colposcopic findings and cervical squamous intraepithelial lesions (SIL) in these matched seropositive and seronegative cohorts.

Methods: A matched prospective study of HIV-seropositive and -seronegative women undergoing cytologic screening, colposcopy, and testing for HPV DNA and other infections at each visit.

Results: 'Twenty-three HIV-seropositive women were matched with 23 seronegative women by cervical cytology reading, lifetime number of sexual partners, age, and follow-up length. Fourteen pairs of these women had follow-up visits every 4 months, for 56 and 53 total visits in seropositive and seronegative women, respectively. After matching, the groups had a similar overall prevalence of HPV DNA and of HPV oncogenic (high risk) types at baseline. On follow up, HIV-seropositive women were more likely than seronegative women to develop SIL (38\% vs. $10 \%)$, less likely to have negative cytology (34\% vs. $60 \%$, overall $P=0.03$ ), more visits with HPV DNA detected (68\% vs. $40 \% P=0.04)$, and more visits with multiple HPV DNA types detected $(18 \%$ vs. $0 \%, P=0.02)$. Colposcopic lesions in the seropositive women were more likely to have sharp borders or mosaicism or to be thick white $(P=0.009)$.

Conclusions: After matching for baseline Papanicolaou smear readings, these data suggest that over time seropositive women have more visits that yield abnormal cytology, more persistent HPV DNA detection, and more colposcopic abnormalities than seronegative women. Infect. Dis. Obstet. Gynecol. 7:158-164, 1999.
\end{abstract}

KEY WORDS

cervical cytology; HIV seropositive; HPV DNA; colposcopy

everal studies have demonstrated an increased $\checkmark$ prevalence of squamous intraepithelial lesions (SIL) in human-immunodeficiency-virus- (HIV) seropositive women. ${ }^{1-5}$ While the natural history of
SIL in HIV-seropositive women has not been well defined, an association between increased prevalence of human papillomavirus (HPV) DNA and HIV has been found in many studies. ${ }^{2,4-9}$. These

Grant sponsor: National Institutes of Health; Grant numbers: AI 12192 and AI 07140.

*Correspondence to: Dr. L.O. Eckert, 325 9th Avenue, Department of OB/GYN, Box 359865, Seattle, WA 98104. E-mail: eckert@u.washington.edu 
studies show an association between HPV DNA detection, HIV serostatus, and the presence or absence of SIL.

While multiple studies show an association between HPV DNA, SIL, and HIV seropositivity, most studies have been cross-sectional. Few studies include matching HIV-seropositive with HIVseronegative women for factors that have been associated with HPV DNA, such as number of sexual partners, age, and cervical SIL. In addition, though published prospective data on SIL in HIV-seropositive women has demonstrated persistence of SIL, ${ }^{10}$ prospective reports on SIL with concurrent HPV DNA detection, comparisons of colposcopic findings, and screening for other vaginal and cervical infections in seropositive women are limited.

The purpose of this study was to examine HPV DNA detection among HIV-seropositive and -seronegative women initially matched for cytologic abnormality, age, and number of sexual partners, and to determine prospectively if cytologic abnormalities or HPV DNA detection differed among these matched women over time. In addition to HPV, colposcopic abnormalities as well as other cervical and vaginal infections were compared between the seropositive and seronegative women.

\section{SUBJECTS AND METHODS}

The HIV-seropositive study group consisted of 23 women referred for gynecologic care between $\mathrm{Au}-$ gust 1992 and July 1994 to the Northwest Family Center, a Seattle-King County Public Health Department clinic established to provide medical care to families affected by HIV. Follow up was possible for 14 of these women, for a total of 56 examinations over the 23 -month study interval. Twenty-three HIV-seronegative women attending the Seattle-King County Sexually Transmitted Diseases Clinic who were enrolled in a previously described study of the etiology and natural history of cervical dysplasia ${ }^{11}$ were matched with the seropositive women for comparison. All seronegative women were available for follow-up evaluation, but to maintain matching, only follow-up visits on the subjects matched with the 14 seropositive women were recorded, for a total of 53 examinations over 24 months. Matching was done by cervical cytology reading at baseline, lifetime number of sexual partners, ( \pm five sexual partners), and age (within 5 years with the exception of one woman) because these factors have been associated with detection of HPV DNA. ${ }^{12-17}$ Additionally, because development or persistence of abnormal cervical cytology was a study endpoint, length of follow-up was also matched. After informed consent was obtained, as approved by the University of Washington Institutional Review Board, each study participant underwent a standardized history and physical examination, including colposcopic examination of the vulva, vagina, and cervix.

The women were initially evaluated and then asked to return every 4 months for repeat evaluation. Culture and wet-mount techniques have been described in detail previously. ${ }^{18}$ At each visit, examination and specimen collection included the following. The lateral vaginal wall was swabbed with a cotton-tipped applicator and placed into 0.2 $\mathrm{mL}$ of physiologic saline solution in a test tube. One drop of this saline wet mount was placed on a slide to evaluate for motile trichomonads and clue cells. A second drop was placed on a slide with a drop of $10 \%$ potassium hydroxide to assess amine odor and presence of hyphae. A Papanicolaou smear was obtained using an ectocervical wooden spatula and an endocervical brush. Each specimen was placed into fixative immediately. Dacron endocervical swabs were used to sample for Neisseria gonorrhoeae culture and herpes simplex virus culture and a cytobrush was used to sample for Chlamydia trachomatis culture. One Dacron swab from the vulva and 2 Dacron swabs from the vaginal sidewall were placed into a $1-\mathrm{mL}$ specimen transport medium (STM; Digene Diagnostics, Silver Spring, MD) for vulvar/vaginal HPV DNA detection. Another Dacron swab was used to sample the ectocervix and endocervical canal and placed into STM for cervical HPV DNA detection.

After collection of the samples, $5 \%$ acetic acid was applied to the cervix, and colposcopy of the cervix was performed and recorded on the standardized form. The cervical colposcopic examinations were recorded onto a circular grid representing the cervix that was divided into 18 sections. Extent of cervical lesion(s) was determined colposcopically by adding the number of sections involved, with a maximum of 18 . Colposcopic lesions(s) were characterized by color, punctation, mosaicism, border (sharp vs. diffuse), and location relative to the squamocolumnar junction. The col- 
poscopic exams were performed by single examiners blinded to HPV DNA results in each population (Investigator L.E. for the seropositive women and investigator C.S. for the seronegative women). At each visit of the seropositive women, blood was drawn for lymphocyte subset determination.

If a Pap smear showed atypia or low-grade SIL (LGSIL), the patient was monitored with repeat Pap smears every 4 months but without cervical biopsy. After one high-grade SIL (HGSIL) Pap smear or after two consecutive LGSIL Pap smears, cervical biopsy was performed. Histologically confirmed SIL was treated by cryotherapy or loop electrosurgical excision procedure (LEEP). If treatment occurred, the patient was no longer eligible for study follow up.

Polymerase chain reaction (PCR) amplification was performed using L1 consensus HPV primers, following previously described procedures. ${ }^{19}$ Products of PCR amplification were blotted onto filters and allowed to hybridize with a generic HPV probe and groups of type-specific oligonucleotide probes: HPV 6 and 11 (low risk), HPV 31, 33, 35, and 39 (intermediate risk) and HPV 16, 18, or 45 (high risk). The PGR reaction mixtures also contained $\beta$-globin primers PC04 and GH20 that were used to prime the synthesis of a fragment of the human $\beta$-globin gene. Amplified samples lacking the $\beta$-globin fragment were considered to have insufficient numbers of eukaryotic cells present for HPV types and were termed unsatisfactory. T-cell subsets were done by flow cytometry using CD4 culture cytostat CD3/T4 antibodies. Statistical analyses appropriate for comparisons of matched data were performed. For dichotomous factors, $\mathrm{McNe}$ mar tests for matched pairs were used, while Wilcoxon signed rank tests were used to evaluate associations among continuous factors. Follow-up visits were analyzed using Kruskal-Wallis test for association.

\section{RESULTS}

Of the $23 \mathrm{HIV}$-seropositive women, the mean CD4 count was 0.406 thou per $\mathrm{mL}$ (range 0-1.132). Fourteen had contracted HIV via heterosexual contact and nine via intravenous drug use. Table 1 compares the baseline characteristics in the HIVseropositive and -seronegative populations. The mean age in the seropositive vs. seronegative women was 31.7 vs. 30.4 years $(P=0.2)$, and me-
TABLE I. Baseline characteristics of HIV-seropositive and HIV-seronegative populations matched for age, number of lifetime sexual partners, and cervical cytology result

\begin{tabular}{|c|c|c|c|}
\hline Characteristic & $\begin{array}{c}\text { HIV+ } \\
n=23 \\
(\%)\end{array}$ & $\begin{array}{c}\text { HIV- } \\
n=23 \\
(\%)\end{array}$ & $P$ value ${ }^{a}$ \\
\hline Age (mean) & 31.7 & 30.4 & 0.2 \\
\hline Nonwhite race & $9(39)$ & $2(9)$ & 0.008 \\
\hline Age of first intercourse & 16.0 & 16.9 & 0.2 \\
\hline Lifetime partners (median) & 15 & 13 & 0.5 \\
\hline Currently smoking & $14(61)$ & $11(48)$ & 0.4 \\
\hline Barrier contraception & $12(52)$ & $8(35)$ & 0.3 \\
\hline Extent of lesion (mean) ${ }^{b}$ & 4.7 & 4.2 & 0.6 \\
\hline Prior C. trachomatis/N. gonorrhoeae & $9(39)$ & $10(43)$ & 0.7 \\
\hline \multicolumn{4}{|l|}{ Current cervical/vaginal infections } \\
\hline C. trachomatis/N. gonorrhoeae ${ }^{c}$ & I (04) & 0 & 0.3 \\
\hline Yeast $^{d}$ & $5(22)$ & $\mathrm{I}(04)$ & 0.1 \\
\hline Bacterial vaginosis $^{d}$ & $4(17)$ & $3(13)$ & 0.7 \\
\hline Herpes simplex virus ${ }^{c}$ & 0 & $\mathrm{I}(04)$ & 0.3 \\
\hline T. vaginalis ${ }^{d}$ & 0 & $2(09)$ & 0.2 \\
\hline \multicolumn{4}{|l|}{ Papanicolaou smear } \\
\hline Negative & $6(26)$ & $6(26)$ & \\
\hline ASCUS & $8(35)$ & $8(35)$ & \\
\hline LGSIL & $8(35)$ & $8(35)$ & \\
\hline HGSIL & I (04) & I (04) & 1.0 \\
\hline \multicolumn{4}{|l|}{ HPV DNA types } \\
\hline Low risk $(6, \mathrm{II})$ & $3(13)$ & $2(9)$ & 0.7 \\
\hline \multicolumn{4}{|l|}{ Intermediate risk } \\
\hline$(31,33,35$, or 39$)$ & $3(13)$ & $7(30)$ & 0.3 \\
\hline High risk $(16,18$, or 45$)$ & $4(17)$ & $5(22)$ & 0.7 \\
\hline Any HPV DNA ${ }^{f}$ & $17(74)$ & $15(65)$ & 0.4 \\
\hline Multiple HPV types & $5(22)$ & $4(17)$ & 0.7 \\
\hline
\end{tabular}

aMcNemar's test for matched pairs for dichotomous factors and Wilcoxon signed rank test for continuous factors.

${ }^{b}$ Colposcopically determined, cervical lesion extent recorded on a grid with a maximum involvement of 18 sections.

'Diagnosis by culture.

dDiagnosis by wet mount.

ASCUS = atypical squamous cells of undetermined significance.

fincludes low, intermediate, high or non-typed HPV DNA, HPV types not exclusive.

dian number of lifetime sexual partners was 15 vs. $13(P=0.5)$ in these two groups, reflecting the baseline matching for these factors. Comparing these matched women, no difference was found in factors that have been associated with HPV DNA detection, such as current smoking or age at first intercourse. The seropositive women were more likely to be of nonwhite race than the seronegative women (39\% versus $9 \%, P=0.008$ ). Among those with colposcopically detected lesions, the extent of lesions seen by colposcopy was no different between the matched HIV-seropositive and -seronegative women (mean of $4.7 / 18$ sections versus $4.2 / 18$ sections, $P=0.6$ ).

A past history of $N$. gonorrhoeae or $C$. trachomatis 
TABLE 2. Colposcopic features of cervical lesions seen initially and in follow up in matched HIV-seropositive and -seronegative women

\begin{tabular}{|c|c|c|c|}
\hline Features & $\begin{array}{c}\text { HIV+ } \\
5 \mathrm{I} \text { total visits } \\
(\%)\end{array}$ & $\begin{array}{c}\text { HIV- } \\
52 \text { total visits } \\
(\%)\end{array}$ & $P$ value $^{a}$ \\
\hline Extent of lesion(s) (mean) ${ }^{b}$ & 4.3 & 4.2 & 0.4 \\
\hline Number of lesions (mean) & 1.6 & 1.2 & 0.07 \\
\hline \multicolumn{4}{|l|}{ Cervical lesions } \\
\hline \multicolumn{4}{|l|}{ Location } \\
\hline On junction & $35 / 49$ (7I) & $31(60)$ & 0.6 \\
\hline Remote from junction & $19 / 49(39)$ & $I I(2 I)$ & 0.3 \\
\hline Mosaicism & $38 / 49(78)$ & $22(42)$ & 0.01 \\
\hline Sharp borders & $28(55)$ & $8(15)$ & 0.003 \\
\hline Thick white color & $9(18)$ & $2(04)$ & 0.06 \\
\hline Mosaic, sharp, or white & $44(86)$ & $25(48)$ & 0.009 \\
\hline
\end{tabular}

"Wilcoxon signed rank test.

${ }^{b}$ Colposcopically determined cervical lesion extent recorded on grid with a maximum involvement of 18 sections.

was reported in a high proportion of both groups: $39 \%$ versus $43 \%$ in the seropositive vs: seronegative women $(P=0.7)$. Current infection with $C$. trachomatis, $N$. gonorrhoeae, herpes simplex virus, or bacterial vaginosis was similar in both groups. Wetmount findings of yeast were more common in the HIV-seropositive women than the seronegative women ( $22 \%$ vs. $4 \%, P=0.1$ ), whereas trichomonads were seen more frequently in the HIVseronegative women ( $9 \%$ vs. $0, P=0.2$ ), though neither reached statistical significance.

In both the HIV-seropositive and -seronegative women studied, 8 of 23 (35\%) had atypia on the initial Pap smear and 8 of 23 (35\%) had LGSIL, reflecting matching for baseline cytologic reading. After this baseline cytologic matching, HPV DNA detection was similar in the seropositive and seronegative women, with a high overall prevalence in both groups (78\% and $69 \%, P=0.5)$ and similar distributions of low-risk, intermediate-risk, highrisk, and multiple HPV types.

Colposcopic examination results are presented in Table 2. The mean extent of cervical lesions seen per visit was 4.3 and 4.2 sections in the seropositive and seronegative women, respectively. The mean number of cervical lesions seen on colposcopic exam was also similar in both groups. (1.6 and 1.2, respectively). Most of the cervical lesions were on the squamocolumnar junction in each group (71\% and $60 \%$, respectively). Mosaicism of lesion(s) was seen in $78 \%$ of visits of the seropositive women vs. $42 \%$ of visits of the seronegative women $(P=0.01)$. Sharp borders were more common in the seropositive group (55\% vs. $15 \%, P=$
0.003 ), as were thick white lesions ( $18 \%$ vs. $4 \%, P$ $=0.06$ ). Combining lesion abnormalities, 44 of 51 $(80 \%)$ visits by seropositive women resulted in colposcopically detected mosaicism, sharp borders, or thick white lesions versus $25(48 \%)$ of 52 visits in seronegative women $(P=0.009)$.

Follow-up results are presented in Table 3 . The HIV-seropositive and -seronegative women had 32 and 30 follow-up visits for analysis, respectively. Despite initial matching by Pap smear, cytologic disparities developed between the two groups during follow up. A negative Pap smear was found in 18 of the 30 follow-up visits $(60 \%)$ by HIVseronegative women compared with 11 of the 32 visits (34\%) by seropositive women, while SIL was seen on 12 of the 32 follow-up smears $(38 \%)$ in the seropositive women vs. 3 of the 30 follow-up smears $(10 \%)$ in the seronegative women (overall, $P=0.03$ ). Additionally, two of these SIL lesions were HGSIL proven by biopsy in seropositive women, and these women were treated. None of the seronegative women developed HGSIL on follow up or required treatment.

Additionally, despite similar prevalences of HPV DNA detected on initial examination in these matched women, on follow up, differences emerged. Of the 28 follow-up visits during which HPV DNA data was available for the seropositive women, 19 of 28 (68\%) revealed HPV DNA vs. 12 of $30(40 \%)$ of the follow-up visits by seronegative women $(P=0.04)$. Multiple HPV DNA types were also detected at the follow-up visits of the seropositive but not the seronegative women ( $18 \%$ vs. $0, P$ = 0.02). Low-, intermediate-, and high-risk HPV 
TABLE 3. Characteristics on follow-up visits of matched HIV-seropositive and HIV-seronegative women

\begin{tabular}{lccc}
\hline & $\begin{array}{c}\text { HIV+ } \\
\text { total visits } \\
(\%)\end{array}$ & $\begin{array}{c}\text { HIV- } \\
\text { total visits } \\
(\%)\end{array}$ & \begin{tabular}{c} 
P value \\
\hline Papanicolaou smear
\end{tabular} \\
Negative & $11(34)$ & $18(60)$ & \\
ASCUS & $9(28)$ & $9(30)$ & $0.03^{\mathrm{a}}$ \\
Any SIL & $12(38)$ & $3(10)$ & $0.04^{\mathrm{b}}$ \\
HPV DNA types & & 0 & $0.4^{\mathrm{b}}$ \\
Low risk (6 or II) & $4 / 28(14)$ & $2(7)$ & $0.8^{\mathrm{b}}$ \\
Intermediate risk (3I, 33, 35, or 39) & $4 / 28(14)$ & $4(13)$ & $0.04^{\mathrm{b}}$ \\
High risk (I6, I8, or 45) & $6 / 28(21)$ & $12(40)$ & $0.02^{\mathrm{b}}$ \\
Any HPV DNA & $19 / 28(68)$ & 0 & $\begin{array}{c}\text { ( } \\
\text { Multiple HPV types }\end{array}$ \\
\hline
\end{tabular}

${ }^{2}$ Overall $P$ for difference between HIV+ and HIV- women by Kruskal-Wallis test for association.

bilcoxon signed rank test.

Includes low, intermediate, high or non-typed HPV DNA; HPV types not exclusive.

DNA types were detected during $14 \%, 14 \%$, and $21 \%$ of follow-up visits by seropositive women, respectively, compared with $0,7 \%$, and $13 \%$ of follow-up visits by seronegative women. Cervical/ vaginal infections with $C$. trachomatis, $N$. gonorrhoeae, yeast, bacterial vaginosis, herpes simplex virus, or Trichomonas vaginalis were not different between the two groups at follow up (data not shown).

\section{DISCUSSION}

This study was designed to evaluate HPV DNA presence in HIV-seropositive and -seronegative women matched by cervical cytology, number of lifetime sexual partners, and age, and to then prospectively monitor and evaluate cytologic abnormalities, colposcopic findings, and HPV DNA detection in these matched pairs of women. The $78 \%$ baseline HPV DNA positivity in the seropositive women is consistent with some studies, ${ }^{4,8,20}$ and higher than other studies. ${ }^{9,21}$ This is not surprising; in this study population where so many women had abnormal cytology results, a high prevalence of HPV DNA detected by a sensitive test such as PCR should be expected. A unique aspect of this study was the matching of the seropositive and seronegative women for risk factors associated with HPV DNA, including cytology, age, and number of sexual partners. Given this matching, the similar prevalences of HPV DNA detected at baseline in these seropositive and seronegative women also is not surprising. This finding is in contrast to other studies where HPV detection was lower in HIVseronegative women. $5,9,21$ However, in previous studies, the seropositive and seronegative women were not matched.

This study also prospectively monitored these HIV-seropositive and-seronegative women. Despite initial matching for cytologic reading, differences emerged upon follow up. The presence of SIL on follow-up cytology was significantly more likely among seropositive women. Conversely, seronegative women had more negative cytologic smears on follow up. This increase in progression or persistence of abnormal Pap smears in seropositive women monitored prospectively is consistent with cross-sectional studies that have demonstrated an increased prevalence of abnormal smears in seropositive women and with one prospective study that also demonstrated increased cytologic abnormalities in seropositive women. ${ }^{10}$ The increase in cytologic abnormalities may be related to $\mathrm{HIV}$ mediated immune suppression.

After matching for cytologic abnormalities, no significant differences in HPV DNA detection were seen on initial examination. However, similar to cytologic findings, differences were present on follow-up evaluations. Any HPV DNA and multiple types of HPV DNA were detected more commonly at the seropositive women's follow-up visits. The fact that in the seropositive women, HPV DNA was persistently detected is also consistent with the findings of multiple cross-sectional studies that have demonstrated increased HPV DNA in seropositive women when compared with seronegative women.

In contrast to a prior colposcopic study that found more extensive and multifocal disease in 
HIV-seropositive women, ${ }^{8}$ the colposcopically determined extent of lesion was not different between the HIV-seropositive and -seronegative women in this study, where the seropositive and seronegative groups were matched for Pap readings. However, a trend toward increased number of lesions was seen in the seropositive women. Both groups had a relatively large amount of the cervix involved in the lesion(s) (mean 4.7/18 sections or $25 \%$ ). This may be because the group with LGSIL initially underwent monitoring rather than early biopsy and treatment.

Studies comparing detailed colposcopic descriptions of cervical lesions between seropositive and seronegative women have not been reported. The standardized physical examination form recorded for each patient visit in this study allowed such a comparison, and differences in lesion characteristics between the seropositive and seronegative women were evident. Cervical lesions with sharp borders, thick white color, and mosaicism are concerning. ${ }^{22}$ These lesion characteristics were seen more commonly in the seropositive women despite initial matching for cytology and are consistent with a greater prevalence of abnormal cytology in the seropositive women. Further prospective studies comparing biopsy correlation with lesion appearance between seropositive and seronegative women may be helpful to guide the clinical decisions regarding appropriate biopsy frequency and timing of treatment in seropositive women.

This study is small, and a high prevalence of cervical and vaginal infections other than HPV was not seen in either group. Although other cervical infections have been suggested as cofactors for activation of latent HPV in the cervix, ${ }^{23}$ larger trials where multivariate analyses are possible would be needed to test for potential independent cofactors in HPV DNA expression.

Prospective studies have demonstrated increased cytologic abnormalities ${ }^{10}$ and increased HPV DNA prevalence ${ }^{24}$ in seropositive women. This study matched subjects for HPV DNA risks factors and also demonstrated increased cytologic abnormalities and HPV DNA detection in seropositive women evaluated prospectively. Additionally, this study demonstrated that colposcopic appearance of cervical lesions is more concerning in seropositive women, consistent with cytologic findings. In women, HIV is an independent risk factor for
SIL. ${ }^{10} \mathrm{HIV}$ has also been associated with anal SIL in men independent of HPV. ${ }^{25}$ Clearly, HIVseropositive women are at increased risk for SIL development, persistence, and recurrence. The reasons for this SIL persistence undoubtedly involve HPV DNA expression, the degree of immunosuppression, and other potential cofactors. The exact clinical utility of HPV DNA detection in monitoring seropositive women who are at increased risk for SIL development and persistence requires further study.

\section{ACKNOWLEDGMENTS}

This study was supported in part by NIH grant AI 12192 and training grant AI 07140 (L.O.E.). The authors wish to thank the supportive staff and patients at the Northwest Family Center who made this study possible.

\section{REFERENCES}

1. Schrager LK, Friedland GH, Maude D, et al. Cervical and vaginal squamous cell abnormalities in women infected with human immunodeficiency virus. J Acquir Immune Defic Syndr 1989;2:570-575.

2. Feingold AR, Vermund SH, Burk RD, et al. Cervical cytologic abnormalities and papillomavirus in women infected with human immunodeficiency virus. J Acquir Immune Defic Syndr 1990;3:896-903.

3. Schäfer A, Friedmann W, Mielke M, Schwartländer B, Koch MA. The increased frequency of cervical dysplasia-neoplasia in women infected with the human immunodeficiency virus is related to the degree of immunosuppression. Am J Obstet Gynecol 1991;164:593-599.

4. Vermund SH, Kelley KF, Klein RS, et al. High risk of human papillomavirus infection and cervical squamous intraepithelial lesions among women with symptomatic human immunodeficiency virus infection. Am J Obstet Gynecol 1991;165:392-400.

5. Laga M, Icenogle JP, Marsella R, et al. Genital papillomavirus infection and cervical dysplasia- opportunistic complications of HIV infection. Int J Cancer 1992;50: $45-48$.

6. Klein RS, Ho GY, Vermund SH, Fleming I, Burk RD. Risk factors for squamous intraepithelial lesions on $\mathrm{Pa}$ panicolaou smear in women at risk for human immunodeficiency virus infection. J Infect Dis 1994;170:1404 1409.

7. Seck AC, Faye MA, Critchlow CW, et al. Cervical intraepithelial neoplasia and human papillomavirus infection among Senegalese women seropositive for HIV-1 or HIV-2 or seronegative for HIV. Int J STD AIDS 1994; 5:189-193.

8. Maiman M, Fruchter RG, Serur E, Remy JC, Feuer G, Boyce J. Human immunodeficiency virus infection and cervical neoplasia. Gynecol Oncol 1990;38:377-382. 
9. Sun XW, Ellerbrock TV, Lungu O, Chaisson MA, Bush TJ, Wright TC Jr. Human papillomavirus infection in human immunodeficiency virus-seropositive women. Obstet Gynecol 1995;85:680-686.

10. Fruchter RG, Maiman MM, Sedlis A, Bartley L, Camilien L, Arrastia CD. Multiple recurrences of cervical intraepithelial neoplasia in women with the human immunodeficiency virus. Obstet Gynecol 1996;87:338344.

11. Koutsky LA, Holmes KK, Critchlow CW, et al. A cohort study of the risk of cervical intraepithelial neoplasia grade 2 or 3 in relation to papillomavirus infection. $\mathrm{N}$ Engl J Med 1992;327:1272-1278.

12. Ley G, Bauer HM, Reingold A, et al. Determinants of genital human papillomavirus infection in young women. J Natl Cancer Inst 1991;83:997-1003.

13. Rosenfeld WD, Vermund SH, Wentz SJ, Burk RD. High prevalence rate of human papillomavirus infection and association with abnormal Papanicolaou smears in sexually active adolescents. Am J Dis Child 1989;143: 1443-1447.

14. Daling JR, Sherman KJ, Weiss NS. Risk factors for condyloma acuminatum in women. Sex Transm Dis 1986; 13:16-18.

15. Villa LL, Franco ELF. Epidemiologic correlates of cervical neoplasia and risk of human papillomavirus infection in asymptomatic women in Brazil. J Natl Cancer Inst 1989;81:332-340.

16. Martinez J, Smith R, Farmer M, et al. High prevalence of genital tract papillomavirus infection in female adolescents. Pediatrics 1988;82:604-608.

17. Kiviat NB, Koutsky LA, Paavonen JA, et al. Prevalence of genital papillomavirus infection among women attending a college student health clinic or a sexually transmitted disease clinic. J Infect Dis 1989;159:293302.

18. Eckert LO, Koutsky LA, Kivat NB, Krone MR, Stevens LE, Eschenbach DA. The inflammatory pap smear: What does it mean? Obstet Gynecol 1995; 86:360-366.

19. Bauer HA, Green CE, Manus MM. Detection of genital HPV infection using PCR. In: Harrington C, McGee J, editors. Diagnostic Molecular Pathology: A Practical Approach. Oxford, England: Oxford University Press, 1992, p 131-152.

20. Gissl'en M, Chiodi F, Fuchs D, et al. Markers of immune stimulation in the cerebrospinal fluid during HIV infection: a longitudinal study. Scand J Infect Dis 1994; 26:523-532.

21. Lafeuillade A, Tamalet C, Pellegrino P, de Micco P, Vignoli C, Quilichini R. Correlation between surrogate markers, viral load, and disease progression in HIV-1 infection. J Acquir Immune Defic Syndr 1994;7:10281033.

22. Burghardt E. Colposcopy and Cervical Pathology. 2nd ed. New York: Thieme Medical Publishers, Inc.; 1991. p 219-221.

23. Vandenveld C, Van Beer D. Risk factors inducing the persistence of high-risk genital papillomaviruses in the normal cervix. J Med Virol 1992;38:226-232.

24. Sun XW, Kuhn L, Ellerbrock TV, et al. Human papillomavirus infection in women infected with human immunodeficiency virus. N Engl J Med 1997;337:13431349.

25. Critchlow CW, Surawicz CM, Holmes KK, et al. Prospective study of high grade anal squamous intraepithelial neoplasia in a cohort of homosexual men: influence of HIV infection, immunosuppression and human papillomavirus infection. AIDS 1995;9:1255-1262. 


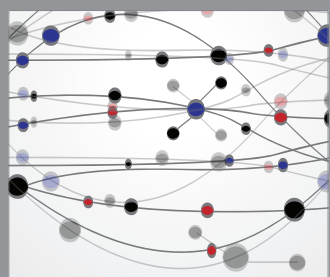

The Scientific World Journal
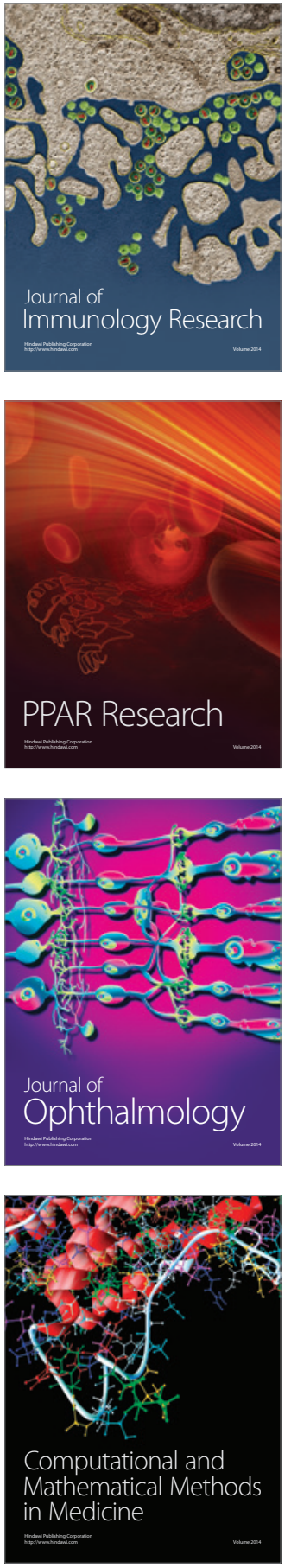

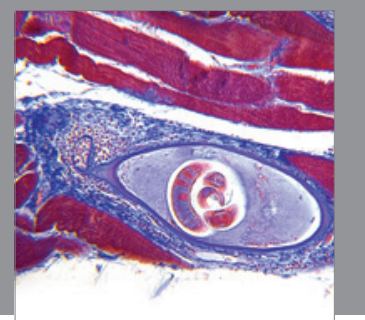

Gastroenterology

Research and Practice
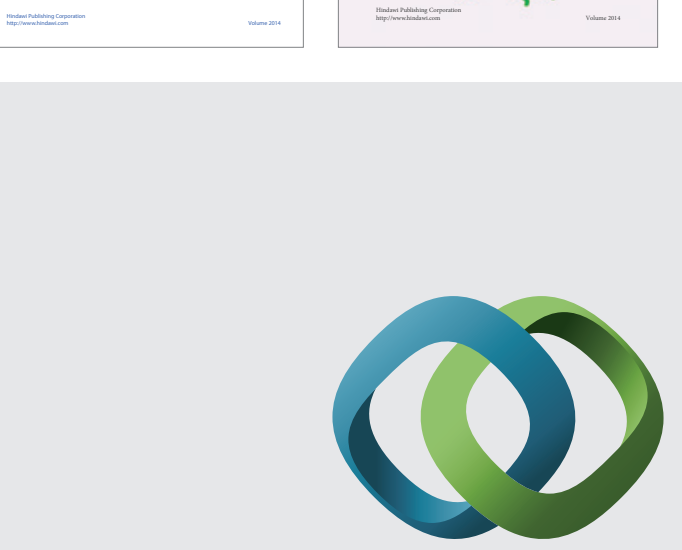

\section{Hindawi}

Submit your manuscripts at

http://www.hindawi.com
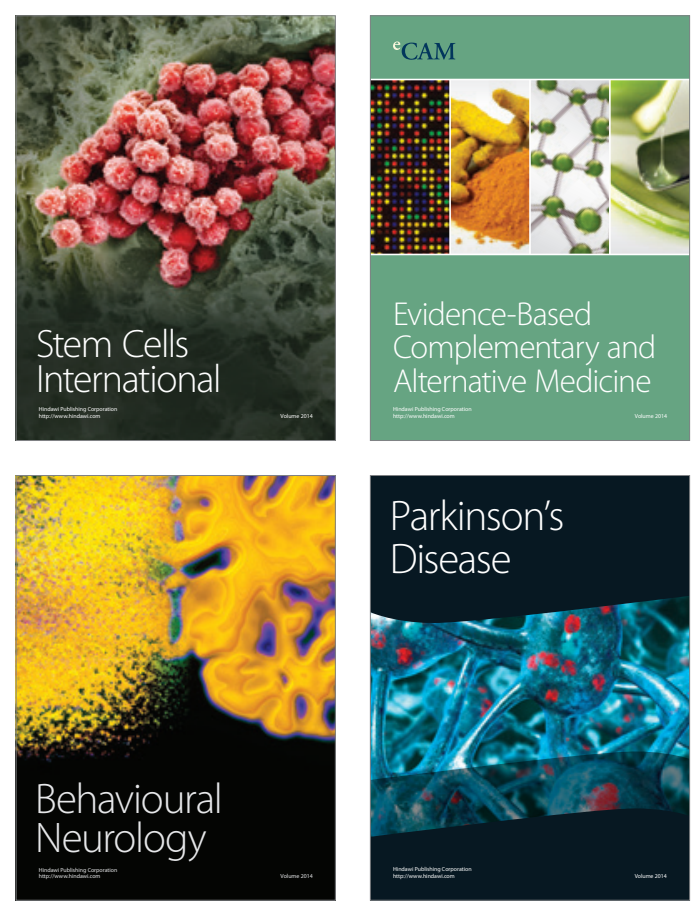

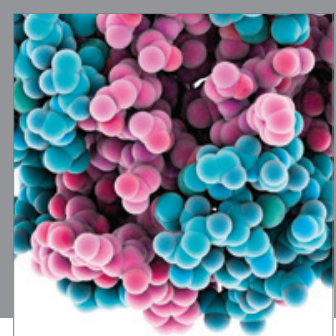

Journal of
Diabetes Research

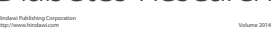

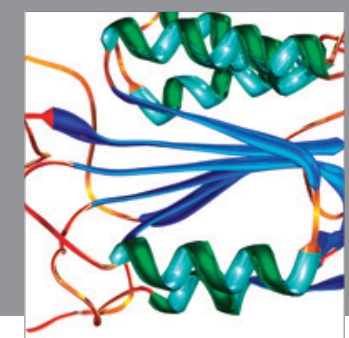

Disease Markers
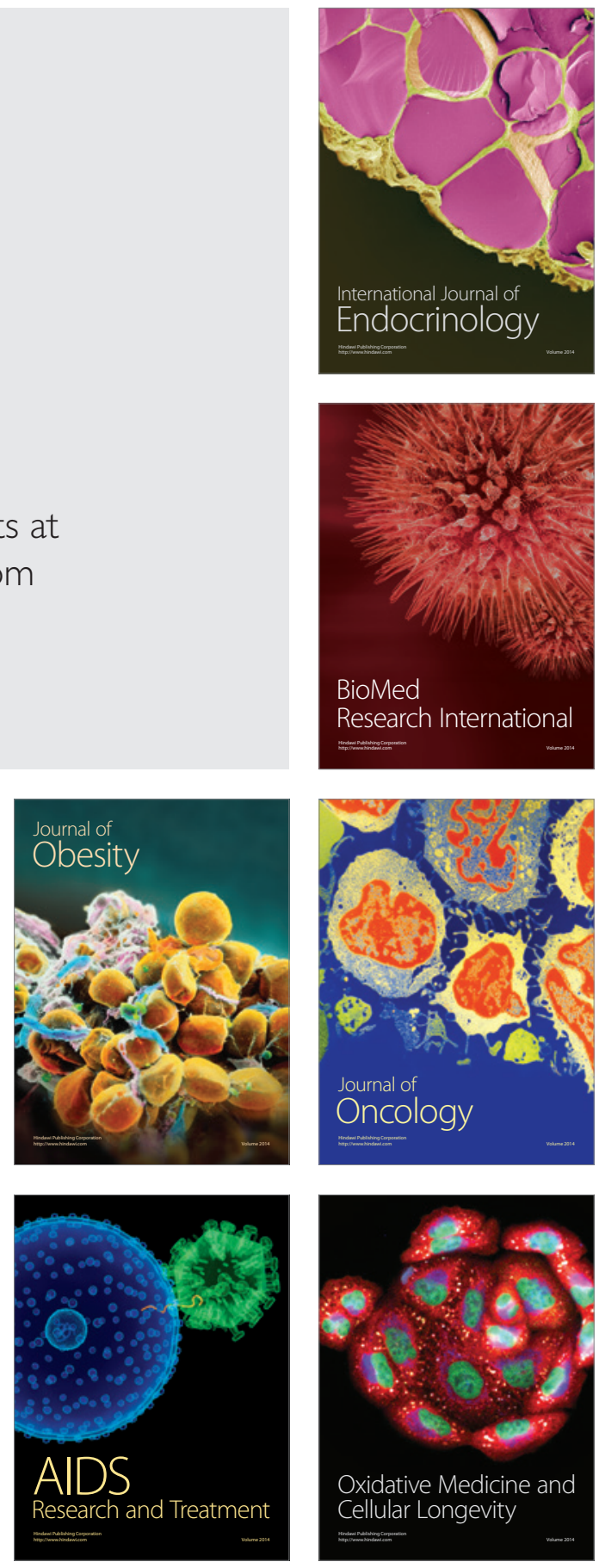